of the persistent pain of vertebral body injury, mainly the aggressive hemangioma, the tumoural processes (myeloma and metastasis) and also in the osteoporotic vertebral collapse. We present our experience in this technique.

Methods We show seven clinical cases, with radiology pictures, about PVP guided by TAC, under local anaesthesia and general sedation, which first indications were to relieve the pain. There are 5 women with an average age of 65 years (40-80) and osteoporotic collapse vertebral. one of them with an hemangioma located in D12 also, a man 72 years old with an hemangioma in L3 and a man 85 years old with a metastasis in L2 secondary to renal tumour.

Results In the seven cases we got to relieve the pain before, the consolidation of the vertebral body, the reduction of the hospitalisation period and saving to have analgesic drugs. In none of them we had complications, except for a case that we caused leakage of cement into adjacent disks, without secondary effects. In the case of the vertebral metastasis the posterior wall was fractured and pushed in the channel, but we didn't cause leakage of cement into the channel.

Conclusion The PVP has been an effective technique in the treatment of the persistent pain in the osteoporotic vertebral collapse, vertebral hemangioma and tumoural process in our patients. It could be an alternative good treatment, and besides it allows an earlier mobilisation, as it prevents the apparition of deformities and possible secundaries complications about this. This is not an expensive procedure, with some possible risk derivated from the technique. Results for long-term follow-up are not yet known and we should make controlled studies to specify which patients could be benefited by technique.

\section{AB0188 BONE MINERAL DENSITY(BMD), SERUM CALCIDIOL AND KNEE OSTEOARTHRITIS}

${ }^{1} \mathrm{M}$ Bonilla Hernán, ${ }^{1} \mathrm{P}$ Aguado, ${ }^{2} \mathrm{~L}$ Vacchiano, ${ }^{3} \mathrm{E}$ Martinez, ${ }^{1} \mathrm{~F}$ Gamero, ${ }^{2} \mathrm{~B}$ Berriatua, ${ }^{1}$ E Martín Mola. ${ }^{1}$ Rheumatology; ${ }^{2}$ General Practisiam; ${ }^{3}$ Biochemistry, Hospital La Paz, Madrid, Spain

\subsection{6/annrheumdis-2001.627}

Background Relationship between knee osteoarthritis (OA) and bone mass has brought a great interest. Serum calcidiol (CS) low level has been associated with a higher incidence and faster progression of OA in elderly women, but this relation has not been defined in young postmenopausal women.

Objectives The aim of the study was to determine the prevalence of knee OA in postmenopausal women and its relationship with bone density and CS levels.

Methods Prospective study realised in 89 women (mean age \pm 5 years: range:47-66), all of them being diagnosed of natural menopause for at least 2 years before they were included in this study.(mean value of $7.9 \pm 2$ ). They were recruited at a rheumatology consultory. CS levels were determined by RIA, along the winter time because of the lest sunny hours during that period. Two critical values were established $(<10$, to calculate deficit prevalence. BMD was measured in lumbar spine and hip with DEXA (Hologic QDR 1000). Patients had to fulfil the WHO criteria to be diagnosed of osteoporosis. Knee OA was defined by a grade $>2$ in Kellgren-Lawrence radiologic scale. Prevalence difference was analysed by contingency tables.

Results A total of 58 women had OP while 31 had normal BMD. SC mean value was $13 \pm 7.1 \mathrm{ng} / \mathrm{ml}$ and there were no significant differences comparing osteoporotic (OP) women with non-OP women. Knee OA prevalence was 65 (73\%). In the OP. group and 80.6 in the non-OP. group. $<10$ and $20 \mathrm{ng} / \mathrm{ml} \mathrm{CS}$ deficit prevalence was $38.7 \%$ and $61.3 \%$ respectively women with knee OA and $39 \%$ and $78.3 \%$ respectively in women without knee OA.

Conclusion No inverse relationship was found between OP and knee OA in our group. CS deficit was not associated with a higher prevalence of knee OA in young postmenopausal women.

\section{AB0189 BONE TURN-OVER AND THE INFLUENCE OF LOW DOSE GLUCOCORTICOSTEROIDS ON BONE MASS IN RHEUMATOID ARTHRITIS PATIENTS}

JK Lacki, P Leszczynski, I Korczowska, P Hrycaj. Rheumatology, University of Medicine, Poznan, Poland

\subsection{6/annrheumdis-2001.628}

\section{Background}

Objectives The present study has been undertaken to evaluate bone turn-over in rheumatoid arthritis (RA) patients as well as the influence of low dose glucocorticosteroids (GCS) on bone mass loss.

Methods Ninety patients with establish RA has been investigated. The patients have been divided into two groups: 44 patients treated with GCS (age 52,5 $\pm 12,4$ years, disease duration 122 \pm 102 months, total dose of GCS, equivalent to prednisone $-7,4$ $\pm 8,3 \mathrm{~g}$ ) and 46 patients who were not treated with GCS (Age $54,3 \pm 9,7$ years, disease duration $134 \pm 120$ month). Fifty patients have been assessed twice (after 12 month). Bone mineral content and bone mineral density have been determined in all patients in distal forearm. Additionally, some biochemical markers of osteoporosis: osteocalcin, alkaline phosphatase-bone formation, carboxyterminal telopeptides of type I collagen (CTx), procollagen type I carboxyterminal propeptide (PICP), deoxypyridynoline and some proinflammatory cytokine: IL-1a, IL-6, TNF-alpha, GM-CSF has been determined. No difference in bone metabolism between RA patients receiving GCS treatment and those treated without GCS was shown.

Results

Conclusion It is concluded that anti-inflammatory effect of GCS may balance the direct effect of GCS on bone mass in RA patients, particularly those with short term treatment.

\section{AB0190 IS BONE MASS AND BONE LOSS RELATED TO LONG- TERM GLUCOCORTICOSTEROIDS IN RHEUMATOID ARTHRITIS AND LUPUS ERYTHEMATOSUS PATIENTS?}

P Leszczynski, I Korczowska, JK Lacki. Department of Rheumatology and Clinical Immunology, University School of Medicine, Poznan, Poland

\subsection{6/annrheumdis-2001.629}

\section{Background}

Objectives The purpose of this study was to determine the correlation between chronic active inflammatory process and longterm glucocorticosteroids theraphy (GCS) and bone loss in patients (pts) with rheumatoid arthritis (RA) and systemic lupus erythematosus (SLE).

Methods We have studied a sample of 128 women (90 RA pts and 38 SLE pts). All SLE pts and 44 RA pts were on chronic GCS (disease duration and duration of GCS therapy was: $108 \pm$ 82 months and $122 \pm 102$ months, respectively). Total dose of GCS was $25.3 \pm 28.19 \mathrm{~g}$ in SLE group and $7.4 \pm 8.3 \mathrm{~g}$ in RA 